\title{
Restoration of functional gait in paraplegic patients with the RGO-II hybrid orthosis. A multicenter controlled study. II: Physiological evaluation
}

\author{
P Thoumie ${ }^{1}$, G Le Claire ${ }^{2}$, J Beillot ${ }^{3}$, J Dassonville ${ }^{3}$, T Chevalier ${ }^{4}$, B Perrouin-Verbe ${ }^{5}$, M Bedoiseau $^{1}$, \\ M Busnel $^{2}$, A Cormerais ${ }^{2}$, A Courtillon ${ }^{6}$, JF Mathe ${ }^{5}$, F Moutet $^{7}, \mathrm{G} \mathrm{Nadeau}^{8}$ and E Tanguy ${ }^{7}$ \\ ${ }^{1}$ SRF Groupe Hospitalier Bichat-Claude-Bernard, 16 Rue Henri-Huchard, 75018 Paris; ${ }^{2}$ CRRF de Kerpape, \\ 56321 Lorient; ${ }^{3}$ Unité de Biologie et Médecine du Sport, Chu Ponchaillou, 35033 Rennes Cedex; ${ }^{4}$ Service de \\ Gastro-Entérologie Groupe Hospitalier Bichat-Claude-Bernard, 75018 Paris; ${ }^{5}$ SRF, CHR-Hopital Saint-Jacques, \\ 44035 Nantes; ${ }^{6}$ CRRF Rennes-Beaulieu, 35700 Rennes; ${ }^{7}$ CRRF Pen-Bron, 44440 La Turballe; ${ }^{8}$ CRRF de \\ l'Arche, 72650 Saint-Saturnin, France
}

\begin{abstract}
We have investigated physiological changes in 21 patients with a spinal cord injury who were fitted with the RGO-II hybrid orthosis. All parameters were measured before and after a training programme in order to evaluate the benefit of gait rehabilitation, cardiovascular adaptation, constipation, spasticity and osteoporosis. A tendency for the improvement in cardiovascular function was noticed, and a segmental decrease in right colonic transit time was observed. However there was no reproducible change in spasticity, and no gain in bone mineral density. These data suggest that the physiological benefits which occur when patients walk with the aid of a hybrid orthosis only correct the effects of immobility. In addition, we did not find any physiological improvement regarding the neurological lesion (spasticity or osteoporosis).
\end{abstract}

Keywords: spinal cord injury; gait; orthosis; osteoporosis; constipation; cardiovascular state

\section{Introduction}

One of the major goals of the rehabilitation of individuals with paraplegia is to prevent acute complications such as pressure sores or urinary tract infections, as well as long term complications such as constipation, spasticity and osteoporosis.

Classical rehabilitation programmes include early standing, which prevents pressure sores and has been proven to correct hypercalciuria related to immobilisation osteoporosis. ${ }^{1}$ However, the long term benefits of standing remain unclear. ${ }^{2}$ Aside from classical rehabilitation and drug therapy, programmes presently offered to paraplegic patients include upper limb exercises to improve cardiovascular function, ${ }^{3}$ or electrical stimulation of lower limbs to reduce spasticity. ${ }^{4}$

The patient's neurological level dictates their success in ambulating with a mechanical orthosis. Typically, paraplegic subjects discontinue ambulating after being discharged from their rehabilitation programme. Additionally, the idea that gait recovery with an orthosis may improve physiological factors has not yet been clearly proved.

Training paraplegic subjects to use the RGO-II hybrid orthosis ${ }^{6}$ gave us the opportunity to evaluate the effects of standing and walking concerning physiological parameters. We expected that selected parameters would improve as a result of standing, upper limb exercises and functional electrical stimulation.
In the first part of this study, we observed patients who were able to stand up and walk with the hybrid orthosis, and we pointed out the limitations: only a few patients continued to use the orthosis after the training programme. It was crucial, therefore, to investigate the potential physiological benefits of walking with the RGO-II in order to evaluate the hybrid orthosis' usefulness as a therapeutic walking system. The purpose of this study was to examine physiological changes in patients who participated in the training programme; specifically, we focused on four parameters: cardiovascular function, spasticity, constipation and osteoporosis.

\section{Subjects and methods}

Subjects

Twenty-one patients from six centers were included in this study between January 1992 and December 1993: one woman and twenty men, mean age: 33 years (range: 20-53), level of lesion: C8-T12, mean time since the onset of paraplegia: 26 months (range 4-72). All subjects successfully completed the training programme in 3-14 months, including 2-h sessions three times a week.

At the beginning and at the end of the training programme we performed clinical evaluations of the 
physiological parameters with one exception: the evaluation of osteoporosis was performed 16 months after the start of the training programme.

All subjects participated in the clinical evaluation of spasticity. Subjects were included in subgroups for physiological evaluation according to their ability to reach the investigation laboratory.

\section{Evaluation}

\section{Cardiovascular function}

Resting heart rate and blood pressure were recorded in all patients. Cardiovascular adaptation to exercise was investigated according to the protocol originally developed by Le Claire, et $\mathrm{al}^{7}$ in 10 patients: one woman and nine men, mean age: 31 years (range: 20-40), level of injury: T2-T10, mean time from onset: 25 months (range: 4-47).

The investigation was performed with an arm crank ergometer (EPC 77701 Rey Thomas, France) while the patient was in a sitting position. After an initial warm-up phase, exercise intensity was increased by 30 -Watt steps ( 2 min of work, 1 min of rest) and the workout continued until the patient reached exhaustion. Heart rate, energy consumption and lactate rate were measured at each level. Energy consumption was automatically recorded with the EOS Sprint Metabographe Jaeger, Germany. Lactate blood concentration was measured with microdosage with the Kontron-LA 640 analyser (Kontron, Switzerland) after obtaining a sample of blood at ear lobe.

All of the parameters were calculated at each step, at the end of the workout, and at 1-min intervals for 3 min after the workout.

\section{Constipation}

Constipation levels were evaluated by means of a radiopaque markers transit study, a classical method for investigating constipation from neurological causes. $^{8}$

Nine patients were tested: one woman and eight men, mean age 31 years (range: $20-44$ ), level of lesion T3-T12, time since the onset of paraplegia: 32 months (range: 10-72). No special diet or medication affecting colonic transit was used in the 2 weeks prior to each evaluation.

We employed a simplified protocol to reduce X-ray exposure: patients ingested a single daily dose of 20 radiopaque markers during 3 days and abdominal radiographs were carried out $24 \mathrm{~h}$ after the last dosage. Then the number of markers in each part of the colon (right and left colon, and the sigmoid) and the total values were counted.

\section{Spasticity}

We used a clinical subjective classification to confirm any positive short term or long term effect of stimulation that might occur in the hours or days after stimulation. Also, 21 patients performed self evaluation with regard to four levels of muscle tone assessed as follows (simplified evaluation derived from the Ashworth score):
Grade 1: No increase in muscle tone

Grade 2: Slight increase in muscle tone without affecting passive limbs movements

Grade 3: Marked increase in muscle tone affecting passive limb movements or requiring drugs

Grade 4: Intractable spasticity

After each session, patients noted changes in muscle tone and the occurrence of spasm or flexion reflex by the hour, so that their observations changed in subsequent days. At the same time, a follow-up control of bowel function and of the skin condition was assessed to eliminate an external reason for changes in spasticity.

\section{Osteoporosis}

Seven patients took part in this study: one woman and six men, mean age: 31 years (range: 26-33), level of injury: T2-T10, mean time from onset: 29 months (range 15-60). The bone mineral density of the lumbar spine and of the femoral neck were assessed by dual photon absorptiometry. The results were calculated in $\mathrm{g} \mathrm{cm}^{-2}$ and then expressed in a relative score that corresponded to the age-control population. The evaluations were performed before training began and 16 months after inclusion in the programme (range: $14-18$ months). All of these patients followed the initial training programme which consisted of three weekly training sessions. At the end of the training programme, they continued to use the orthosis at home (community walkers), or as outpatients (exercise walkers) with the same frequency, as was assessed with monthly supervision.

\section{Statistical analysis}

We conducted a statistical comparison between the values registered before and after training using a variance analysis (ANOVA). An alpha level of 0.05 was considered to be statistically significant.

\section{Results}

\section{Cardiovascular function}

When the patients were at rest, no changes were observed in the clinical parameters of heart rate and blood pressure. We observed the following changes in the patients' cardiovascular adaptation to exercise after the training programme:

- During exercise with increased power steps, the heart rate was slower (Figure 1). Changes were significant at submaximal level:

$$
\begin{aligned}
& \text { at } 0 \text { Watt, } 100 \pm 17 \mathrm{bpm} \text { vs } 113 \pm 16 \mathrm{bpm} \\
& \quad(F=5.82, P<0.05) ; \\
& \text { at } 30 \text { Watts, } \mathrm{HR}=100 \pm 15 \mathrm{bpm} v s 119 \pm 18 \mathrm{bpm} \\
& \quad(F=15.78, P<0.01) ; \\
& \text { at } 60 \text { Watts, } \mathrm{HR}=117 \pm 15 \mathrm{bpm} v s 133 \pm 18 \mathrm{bpm} \\
& \quad(F=16.87, P<0.01) ; \\
& \text { at } 90 \text { Watts, } \mathrm{HR}=142 \pm 15 \mathrm{bpm} v s 163 \pm 18 \mathrm{bpm} \\
& \quad(F=12.55, P<0.01) ; \\
& \text { at } 120 \text { Watts, HR }=168 \pm 9 \mathrm{bpm} v s 177 \pm 12 \mathrm{bpm} \\
& \quad(F=6.28, P<0.05) ;
\end{aligned}
$$




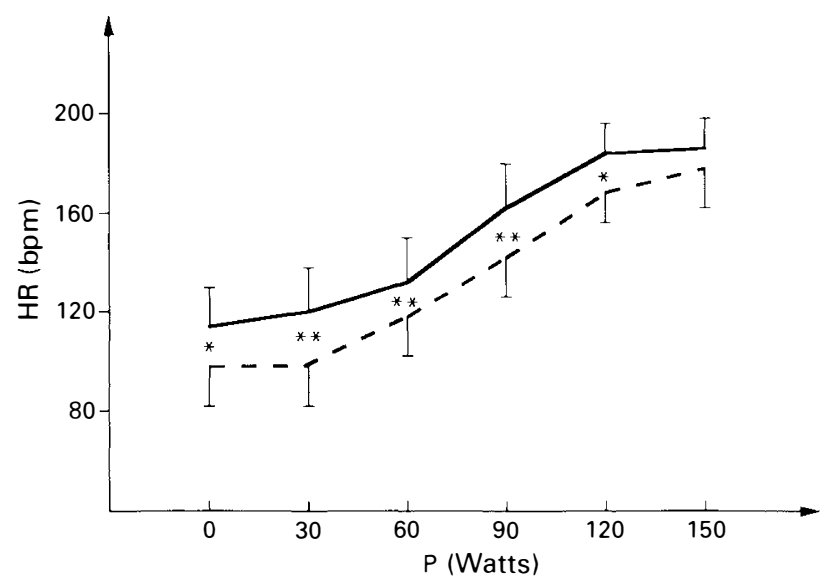

Figure 1 Heart rate changes related to power level during exercise with the cycloergometer $(n=10)$.

Before training sessions:

After training sessions: -.........

${ }^{*}$ and ${ }^{* *}$ : Statistically significant (ANOVA). ${ }^{*}: P<0.05$, **: $P<0.01)$

differences were not significant at the 150-Watt level.

- During exercise at maximal power with the arm crank ergometer (Figure 2), no change was detected in the heart rate value at rest $(79 \pm 20 \mathrm{bpm} v s$ $82 \pm 19 \mathrm{bpm})$ or in the maximal rate $(169 \pm 16 \mathrm{bpm}$ vs $175 \pm 14 \mathrm{bpm})$. Changes in recovery after exercise were not statistically significant: $1 \mathrm{~min}$ afterwards, $\mathrm{HR}=131 \pm 18 \mathrm{bpm}$ vs $139 \pm 19 \mathrm{bpm}$; at $2 \mathrm{~min}, \mathrm{HR}=121 \pm 28 \mathrm{bpm}$ vs $133 \pm 24 \mathrm{bpm}$; at $3 \mathrm{~min}, \mathrm{HR}=114 \pm 22 \mathrm{bpm} v s 122 \pm 22 \mathrm{bpm}$ ).

- Oxygen consumption (Figure 3) was lower for power levels up to 90 Watts, but changes were not significant.

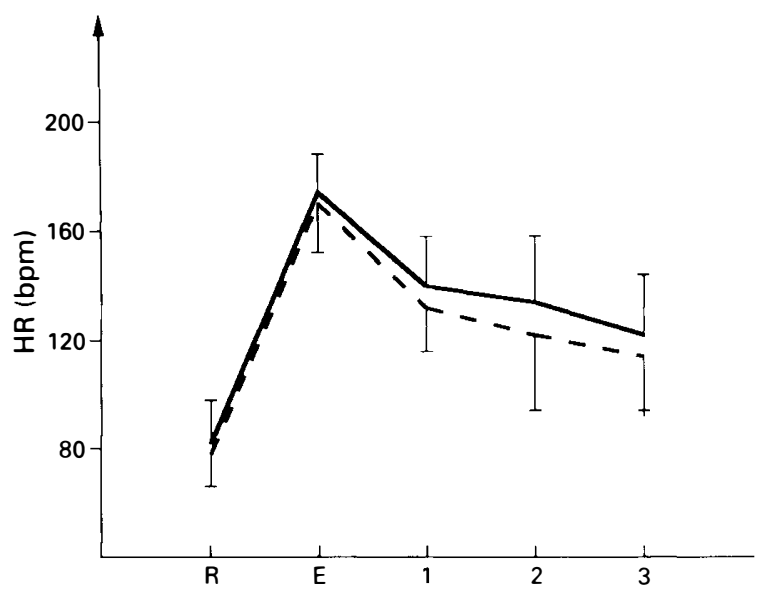

Figure 2 Heart rate changes during workout with the cycloergometer $(n=10)$.

Before training sessions:

After training sessions: -...........

$\mathrm{R}$ : rest; E: exhaustion

1, 2, 3: Recovery time (min)

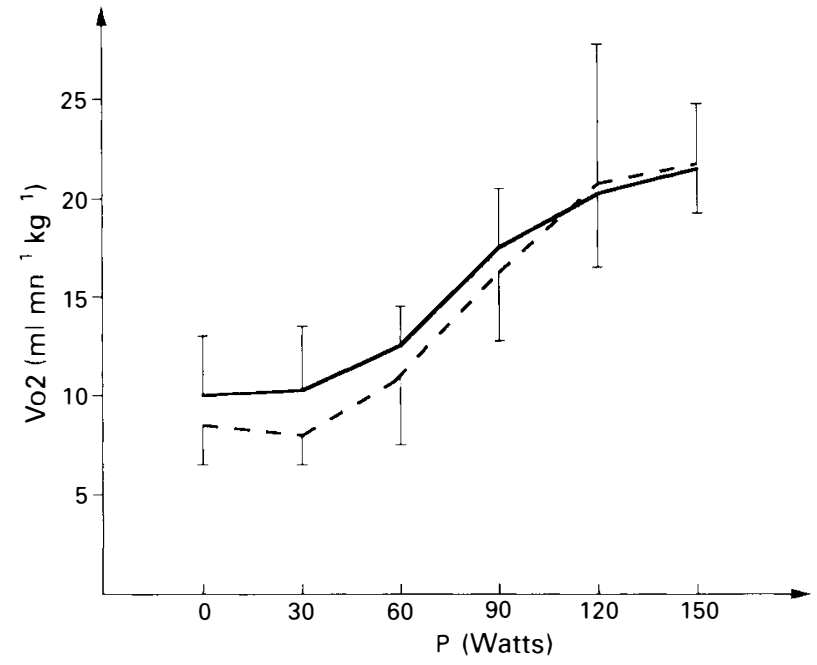

Figure $3 \mathrm{Vo}_{2}$ changes related to power level during exercise with the cycloergometer $(n=10)$.

Before training sessions:

After training sessions: - . . . . . . .

- Lactate blood concentration (Figure 4) was lower for power level up to 120 Watts but only values recorded at the 60-Watt level decreased significantly: $1.63 \pm 0.6 \mathrm{mmoll}^{-1}$ vs $2.18 \pm 0.85 \mathrm{mmoll}^{-1}$ $(F=5.76, P<0.05)$.

\section{Constipation}

The results are presented in Table 1 . The total number of radiopaque markers was significantly different after the training programme: $50 \pm 7 v s 55 \pm 3$. $(F=4.92$, $P=0.05)$. The segmental colonic study showed that a

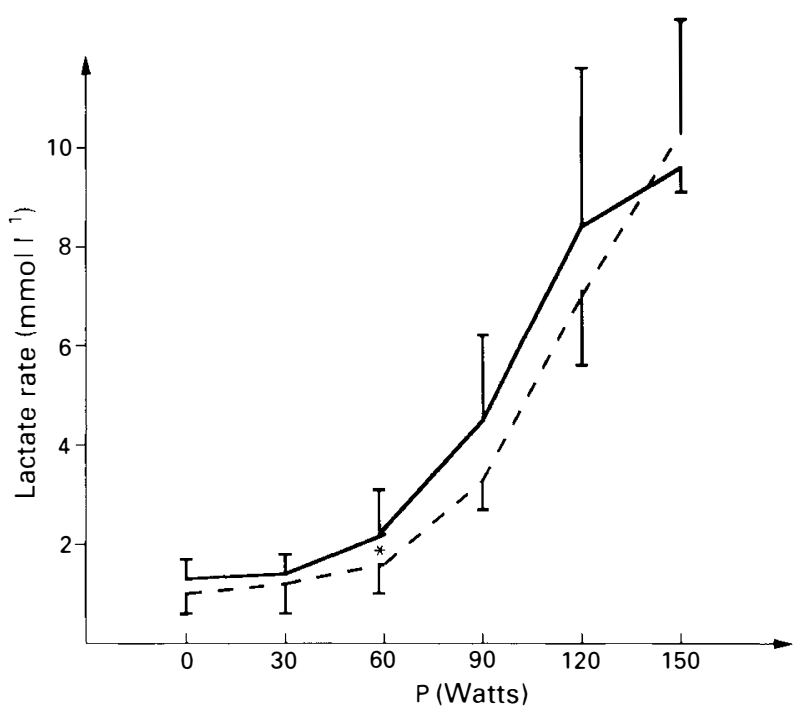

Figure 4 Lactate blood rate changes related to power level during exercise with the cycloergometer $(n=10)$.

Before training sessions:

After training sessions: -..........

*Statistically significant (ANOVA) $P<0.05$ 
Table 1 Number of radio-opaque markers in each region of the colon before and after the training programme (see protocol details in text). Significant changes concern total markers $(P=0.05)$ and right colon markers $(P=0.03)$.

\begin{tabular}{lrrrr}
\hline Name & $\begin{array}{c}\text { Right } \\
\text { colon }\end{array}$ & $\begin{array}{c}\text { Left } \\
\text { colon }\end{array}$ & $\begin{array}{c}\text { Recto- } \\
\text { sigmoid }\end{array}$ & Total \\
\hline Before training & $\begin{array}{r}\text { programme } \\
1\end{array}$ & & & \\
2 & 24 & 20 & 14 & 58 \\
3 & 33 & 26 & 0 & 59 \\
4 & 39 & 16 & 1 & 56 \\
5 & 34 & 12 & 11 & 57 \\
6 & 19 & 20 & 18 & 57 \\
7 & 9 & 21 & 23 & 53 \\
8 & 44 & 5 & 1 & 50 \\
9 & 18 & 4 & 17 & 49 \\
m & 35 & 7 & 15 & 57 \\
SD & 28 & 15 & 11 & 55 \\
After training programme & & 8 & 3 \\
1 & 29 & 4 & 16 & 49 \\
2 & 8 & 14 & 23 & 55 \\
3 & 21 & 18 & 16 & 55 \\
4 & 33 & 9 & 13 & 55 \\
5 & 19 & 34 & 7 & 60 \\
6 & 6 & 21 & 13 & 40 \\
7 & 6 & 18 & 27 & 51 \\
8 & 20 & 8 & 14 & 41 \\
9 & 13 & 15 & 13 & 42 \\
m & 17 & 16 & 16 & 50 \\
SD & 9 & 8 & 6 & 7 \\
\hline & & & &
\end{tabular}

significant decrease in markers occurred in the right colon: $17 \pm 9$ vs $28 \pm 11(F=6.34, P=0.03)$.

\section{Spasticity}

The spasticity level was grade 2 for 16 patients and grade 3 for five patients. Among 16 patients with grade 2 , spasticity remained unchanged in eight patients, decreased to grade 1 in six patients and increased to grade 3 in two patients. Among five patients with grade 3 , spasticity increased to grade 4 in two cases, remained equal in two cases and decreased to grade 2 in one case. FES-induced changes in spasticity were only short term effects: spasticity decreased for seven patients in $0.5-5 \mathrm{~h}$ and increased for four patients in 0.5 to $1 \mathrm{~h}$. No long term effects were observed.

\section{Osteoporosis}

The bone mineral density scores, related to age-control population score, are shown in Figure 5. Compared to normal values, no significant changes were observed at the lumbar level, but a significant decrease in values was seen at the femoral neck level. After the training programme, we found significant decreased values (change $>0.03)$ for four patients, $(0.76 v s 0.80,0.69 v s$ $0.74,0.62$ vs 0.75 and 0.39 vs 0.64 ), increased value for one patient $(0.71 v s 0.65)$, and no significant changes for two patients. $(0.70$ vs 0.73 and 0.63 vs 0.60$)$.
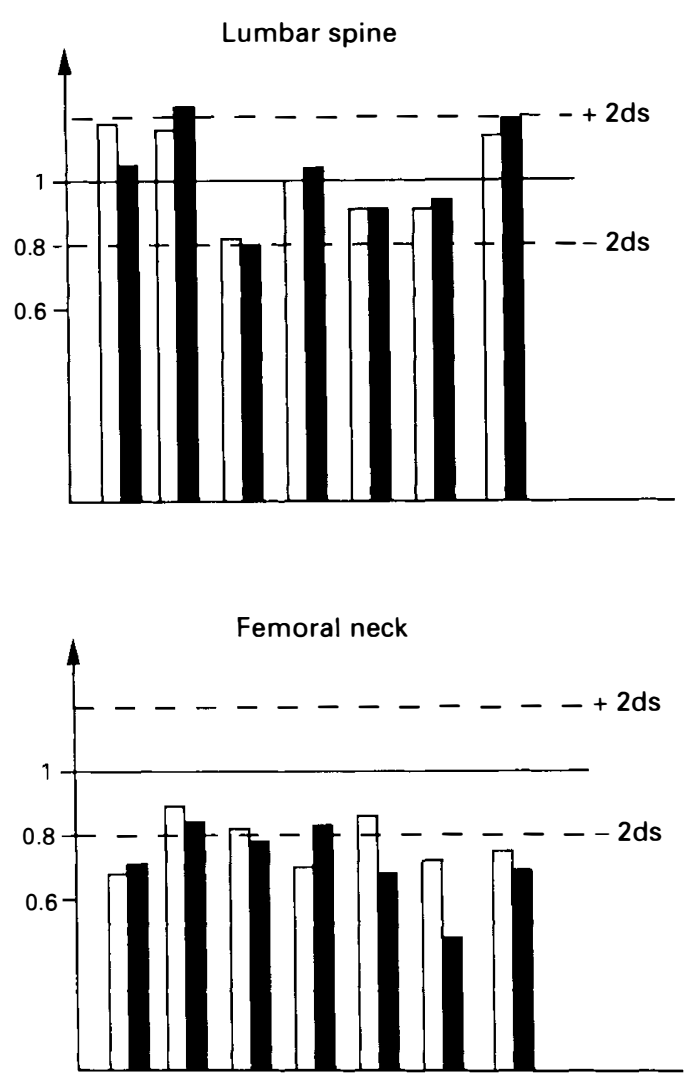

Figure 5 Bone mineral density evaluation in the spinal and the femoral neck regions before $(\square)$ and after $(\square)$ the training programme $(n=7)$. The results are related to age-control population $(Z$-score, with reference value $(=1)$ and standard deviations)

\section{Discussion}

Analysis of results

Cardiovascular fitness decreases in most patients after spinal cord injury. ${ }^{3,9}$ Reconditioning has been proposed to prevent adverse long term effects and to decrease cardiovascular morbidity. In our study, physical conditioning produced a better cardiovascular response to exercise at low or medium power without changes in VO2Max. This suggests that a training programme with the gait orthosis may be considered as an alternative to cardiovascular fitness restoration. Cardiovascular adaptation to upper limb and trunk work seems to comprise the majority of these changes as reported in arm crank ergometry. ${ }^{10}$ But benefits may also result from electrical stimulation of spastic limbs with increased muscle bulk, as Phillips et al proved when using bicycle ergometry. ${ }^{11}$

Constipation occurs in almost all patients after spinal cord injury. Both increased colonic transit time and the loss of anorectal motility may cause constipation in these patients. ${ }^{8}$ Confinement to bed remains a contributing cause of constipation no matter what its origin may be, but even paraplegic patients who can assume a prolonged sitting position complain of long term constipation despite an adapted dietary regime or 
drugs. No studies have previously examined the possibility of easing constipation through weight-bearing or exercise, since standing in a frame is the usual recommendation for improving the physiological status of paraplegics.

The decreased number of radiopaque markers remaining in the right colon after training may correspond to a segmental decrease in the right colon's transit time. The limited effect on the right colon may be ascribed to the colonic innervation since extrinsic innervation of the right colon is not affected by spinal cord lesions. ${ }^{12}$ Weight-bearing (decreased value of transit time in standing $v s$ sitting position during training) and the solicitation of abdominal muscles during gait in low thoracic level paraplegic people may help to produce this change. The absence of change in rectal evacuation and the primary localisation of reduced transit time in the left colon may explain why patients did not notice any changes at the end of training programme.

Spasticity reduction is one goal of rehabilitation for spinal cord injured patients when their comfort, sitting position in wheelchair or daily activities are affected. Customary treatment for this problem includes classical rehabilitation (passive mobilisation) and oral drugs.

FES has long been proposed as a treatment option, but controlled studies have failed to show any reproducible beneficial effect of FES for paraplegic patients. In our study, only seven subjects out of 21 felt that such improvement resulted from FES. Two parameters may be discussed with regard to spasticity changes induced by the RGO-II training programme: the roles of standing and of stimulation.

Few reports have examined the effects that standing produces on spasticity. In 1993, Kunkel et $a^{2}$ evaluated the effects on spasticity for six paraplegic patients when they stood in a frame; and during 5 months of daily sessions, no clinical or neurophysiological changes were observed.

Conversely, a number of studies have sought to evaluate the beneficial effects of stimulation on spasticity. Initial studies reported that clinical improvement lasted from a few hours ${ }^{13}$ to 1 day ${ }^{14}$ following muscular stimulation of spastic muscles. When Bajd et al used the pendulum test to evaluate spasticity, ${ }^{4}$ they found that spasticity decreased in three patients out of six after dermatome stimulation without muscle contraction. More recently, Robinson et al observed favourable short term effects of one 20-min session of muscular stimulation which was assessed with the pendulum test ${ }^{15}$ however, adverse long term effects such as increased spasticity resulted from daily sessions, particularly in those with incomplete paraplegia. ${ }^{16}$ Regarding reduction of spasticity, the RGO-II orthosis does not appear to offer reproducible benefits.

Osteoporosis and increased calcium excretion have been studied largely by Chantraine. ${ }^{17}$ Bone mass loss increases $6-10 \%$ in the first weeks following injury and can range $30 \% 17$ months later, while common activities increase the risk of bone fracture. Weightbearing with a tilt-table has been proven to reduce calcium excretion; even more significant effects have been observed in the first 6 months following injury, ${ }^{1}$ and early standing in a frame actually remains the major preventative treatment for hypercalciuria in paraplegic subjects.

Regarding constituted osteoporosis, however, recent studies have failed to detect any improvement in bone density resulting from rehabilitation programmes which include prolonged standing sessions, ${ }^{2,18}$ gait restoration with a mechanical orthosis ${ }^{5}$ or electrical stimulation with bicycle ergometry. ${ }^{19}$ Our results concur with previous reported data. We found that physical exercises, including weight-bearing and muscular stimulation, do not correct osteoporosis in paraplegic patients. These results correspond to the idea that physiological changes from a neurological cause play a greater role in promoting osteoporosis than does immobility.

\section{Perspectives and conclusion}

In this study, we have observed the physiological changes which spinal cord injured patients underwent after completing gait training. Beyond that, we investigated the complications that resulted from neurological changes and the inactive paraplegic status. Our data clearly indicated that gait restoration only induces changes in cardiovascular and right colonic functions; no change was observed in spasticity, or in osteoporosis. The main question was whether these parameters were unaffected by exercise (they could be related only to neurological changes), or if gait restoration was ineffective because patients did not use the orthosis often enough during a sufficient period of time. Regarding the orthosis' effectiveness, two parameters may be taken into account: the frequency of use and the delay from the onset of paraplegia. Concerning the first point, it may be considered that when ordinary usage of $2-3 \mathrm{~h}$ three times per week is sufficient to induce cardiovascular and digestive changes, this usage may be insufficient to result in changes of osteoporosis. However, in another study ${ }^{5}$ no change in bone mineral density occurred when patients used the RGO mechanical orthosis three times daily, 5 days per week for 18 months. In our study, the addition of FES in the hybrid orthosis did not appear to change these results.

As a means of reducing osteoporosis, we may consider the delay from the onset of paraplegia and orthosis usage in the early phase of rehabilitation. Chantraine ${ }^{17}$ clearly demonstrated that major changes in bone mass occur in the first few weeks following spinal cord injury, and Kaplan et al ${ }^{1}$ showed that weight-bearing reduces hypercalciuria more efficiently in the first few months following injury. Beyond this time period, no therapy has improved bone mineral density., ${ }^{2,5,19}$ Although our study showed that gait restoration did not improve bone density, it is not possible to dismiss the potential effect of gait restoration on calcium metabolism when it has been instituted soon after injury.

Finally, the question of the long term effects of the programme remains unresolved. As Petrosky et $a l^{20}$ 
have pointed out, all of the physiological benefits of the rehabilitation of paraplegic patients stem from regular exercise, and thus no definitive benefit can be expected once training ends. In other words, the indication of the hybrid orthosis for physiological improvement may not be established until others parameters, mainly physical and psychological, permit frequent and prolonged use.

\section{Acknowledgements}

Special thanks are extended to Noelle Ponthieu for improving the English. This work was supported by a grant from the DELTA-7 association.

\section{References}

1 Kaplan PE et al. Reduction of hypercalciuria in tetraplegia after weight-bearing and strengthening exercises. Paraplegia 1981; 19: $289-293$

2 Kunkel CF et al. Effects of standing on spasticity, contracture and osteoporosis in paralyzed males. Arch Phys Med Rehabil 1993; 74: 73-78

3 Nilsson S, Staff PH, Pruet EDR. Physical work capacity and the effect of training on subjects with long-standing paraplegia. Scand J Rehabil Med 1975; 7: 51-56.

4 Bajd T, Gregoric M, Vodovnik L, Benko H. Electrical stimulation in treating spasticity resulting from spinal cord injury. Arch Phys Med Rehabil 1985; 66: 515-517.

5 Ogilvie C, Bowker P, Rowley DI. The physiological benefit of paraplegic orthotically aided walking. Paraplegia 1993; 31: 111-115.

6 Solomonow M et al. The RGO generation II: Muscle stimulation powered orthosis as a practical walking system for thoracic paraplegics. Orthopedics 1989; 12: 1309-1315.

7 Le Claire G et al. Aspects bioénergétiques de l'adaptation cardio-vasculaire chez le paraplégique appareillé en orthèse hybride RGO-II. Résultats préliminaires. In: Actualités en
Rééducation Fonctionnelle et Réadaptation, 17ème série. Masson: Paris, pp 319-329.

8 Beuret-Blanquart $\mathrm{F}$ et al. Colonic transit time and anorectal manometric anomalies in 19 patients with complete transection of the spinal cord. J Auton Nerv Syst 1990; 30: 199-208.

9 Flandrois $\mathrm{R}$ et al. Aerobic performance capacity in paraplegic subjects. Eur J Appl Physiol 1986; 55: 604-609.

10 Drory $\mathrm{Y}$ et al. Arm crank ergometry in chronic spinal cord injured patients. Arch Phys Med Rehabil 1990; 71: 389-392.

11 Phillips CA, Petrofsky JS, Hendershot DM, Stafford D. Functional electrical exercise: comprehensive approach for physical conditioning of spinal cord injured patients. Orthopedics 1984; 7: 1112-1123.

12 Devroede G, Lamarche J. Functional importance of extrinsic parasympathetic innervation of the distal colon and rectum in man. Gastroenterology 1974; 66: 273-280.

13 Lee WJ, McGovern JP, Duval EN. Continuous tetanizing (low voltage) currents for relief of spasm. Arch Phys Med Rehabil 1950; 31: 766-770.

14 Newman LB, Arieff AJ, Wassermann RR. Present status in management of spasticity and spasm: preliminary report. Arch Phys Med Rehabil 1954; 25: 427-436.

15 Robinson CJ, Kett NA, Bolam JM. Spasticity in spinal cord injured patients: 1: Short-term effects of surface electrical stimulation. Arch Phys Med Rehabil 1988; 69: 598-604.

16 Robinson CJ, Kett NA, Bolam JM. Spasticity in spinal cord injured patients: 2: Initial measures and long-term effects of surface electrical stimulation. Arch Phys Med Rehabil 1988; 69: $862-868$.

17 Chantraine A. Clinical investigation of bone metabolism in spinal cord lesions. Paraplegia 1971; 8: 253-259.

18 Biering-Sorensen F, Bohr H, Schaadt O. Bone mineral content of the lumbar spine and lower extremities, years after spinal cord lesion. Paraplegia 1988; 26: 147-149.

19 Leeds EM et al. Bone mineral density after bicycle ergometry training. Arch Phys Med Rehabil 1990; 71: 207-209.

20 Petrofsky JS, Stacy R. The effects of training on endurance and the cardiovascular responses of individuals with paraplegia during dynamic exercise induced by functional electrical stimulation. Eur J Appl Physiol 1992; 64: 487-492. 